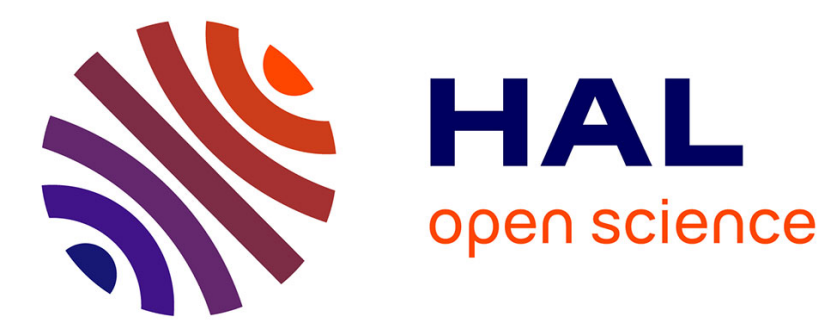

\title{
Can Camera Motions Improve the Perception of Traveled Distance in Virtual Environments?
}

Léo Terziman, Anatole Lécuyer, Sébastien Hillaire, Jan Malte Wiener

\section{To cite this version:}

Léo Terziman, Anatole Lécuyer, Sébastien Hillaire, Jan Malte Wiener. Can Camera Motions Improve the Perception of Traveled Distance in Virtual Environments?. Virtual Reality 2009

- IEEE Annual International Symposium, Mar 2009, Los Angeles, United States. pp.131-134, 10.1109/VR.2009.4811012 . inria-00449796

\section{HAL Id: inria-00449796 \\ https://hal.inria.fr/inria-00449796}

Submitted on 22 Jan 2010

HAL is a multi-disciplinary open access archive for the deposit and dissemination of scientific research documents, whether they are published or not. The documents may come from teaching and research institutions in France or abroad, or from public or private research centers.
L'archive ouverte pluridisciplinaire HAL, est destinée au dépôt et à la diffusion de documents scientifiques de niveau recherche, publiés ou non, émanant des établissements d'enseignement et de recherche français ou étrangers, des laboratoires publics ou privés. 


\section{Can Camera Motions Improve the Perception of Traveled Distance in Virtual Environments?}

\author{
Léo Terziman* \\ Bunraku Team \\ INSA/INRIA \\ France
}

\author{
Sébastien Hillaire \\ Bunraku Team \\ Orange Labs/INRIA/INSA \\ France
}

\author{
Jan M. Wiener $§$ \\ Center for Cognitive Science \\ Freiburg University \\ Germany
}

\begin{abstract}
This paper reports one experiment conducted to evaluate the influence of oscillating camera motions on the perception of traveled distances in virtual environments. In the experiment, participants viewed visual projections of translations along straight paths. They were then asked to reproduce the traveled distance during a navigation phase using keyboard keys. Each participant had to complete the task (1) with linear camera motion, and (2) with oscillating camera motion that simulates the visual flow generated by natural human walking. Taken together, our preliminary results suggest that oscillating camera motions allow a more accurate distance reproduction for short traveled distances.
\end{abstract}

Keywords: camera motion, walking, perception, distance, firstperson-navigation

Index Terms: H.5.1 [Information Interfaces and Presentation]: Multimedia Information Systems-Artificial, augmented, and virtual realities; H.5.2 [Information Interfaces and Presentation]: User Interfaces-Input devices and strategies, Interaction styles, Usercentered design; I.3.7 [Computer Graphics]: Three-Dimensional Graphics and Realism-Virtual reality

\section{INTRODUCTION}

Navigation is one of the main interaction tasks in Virtual Environments (VE). In the real world, different senses contribute to the sensation of walking: the vestibular sense, the proprioceptive sense and the efferent copies of the muscular commands [1]. However, most applications of Virtual Reality (VR) cannot afford expensive locomotion interfaces that would provide users with vestibular and proprioceptive cues. Even if passive physical motion appears to be a more effective cue, Harris et al. [2] showed that visual flow also influences the perception of self-motion.

Thus, visual simulations of human walking have been developed, in particular with the use of camera shifts calculated to reproduce the motions of the eyes of a human being during the walk, in response to the oscillations of the user's head produced at each step by natural walking [3][5]. Lécuyer et al. [5] proposed a compensated oscillating camera motion which automatically modifies the angle of the camera in order to keep a fixed point as the focus point of the camera. Using subjective questionnaires they found that oscillating motions were preferred by the participants over the "classical" linear motions. In a second survey, they found that compensated oscillating camera motion was also preferred by the participants over the regular oscillating camera motion.

Hillaire et al. [3] proposed to use an eye-tracking system in order to improve the use of compensated oscillating camera motions.

\footnotetext{
*e-mail: leo.terziman@irisa.fr

†e-mail: anatole.lecuyer@irisa.fr

† e-mail: sebastien.hillaire@irisa.fr

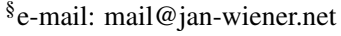

IEEE Virtual Reality 2009

14-18 March, Lafayette, Louisiana, USA

978-1-4244-3943-0/09/\$25.00 @2009 IEEE
They dynamically adapted the focus point of the camera to match the participants' gaze. By this means they were able to simulate a more realistic camera motion. Still using questionnaires they found that participants have globally preferred the compensated camera motion adapted with the eye-tracking system.

However, as for today, camera motions still lack formal evaluations of their intrinsic properties. For instance, what is the influence of using this type of camera motion on the perception of properties of VE, such as the perception of traveled distances, the sense of orientation, or the perception of scale?

In the remainder of this paper, we will focus on the effect of compensated oscillating camera motions on the perception of traveled distances in virtual environments.

\section{EXPERIMENT}

The goal of this experiment is to investigate if oscillating camera motions result in an increased accuracy of traveled distance perception in Virtual Environments (VE).

VE are often used in experiments that involve path integration or wayfinding. Indeed, VE enable to control more precisely the parameters of the experiment. For example, Meilinger et al. [6] used a photorealistic virtual environment representing a medieval city in order to study the influence of a set of different distracting tasks on the working memory task involved in memorizing a recently experienced route. Also with the help of VE, Riecke et al. [9] found that, in absence of vestibular and proprioceptive cues, visual flow information alone was sufficient to support path integration for simple navigations, composed of one or two segments. Using VE, some studies could even demonstrate an increase in path integration accuracy for more complex paths [11]. On the other hand, it appears that VR, lacking proprioceptive and vestibular sensations, can also result in systematic misperceptions of the general form of traveled paths [10].

In our experiment, participants were presented with visual projections of a straight path. For half of the trials, the camera moved linearly (as in a car simulation), and for the remaining trials the camera oscillates around three axes in addition to the linear movement in order to simulate visual flow generated by human walking. We used the same compensated oscillating camera motions as proposed in [5] and [3]. The participants' task was to estimate and reproduce the traveled distance.

\subsection{Methods}

\subsubsection{Participants}

Twelve participants aged between 22 and 59 (mean=30, standard deviation=12.6) performed this experiment. There were 8 men and 4 women. One participant was left-handed, all the other participants were right-handed. All participants were naive with respect to the experimental setup and to the purpose of the experiment.

\subsubsection{Set-up}

The experiment was carried out in a darkened room. Participants sat 3 meters in front of a half-cylindrical projection screen of 9.5 meters by 2.4 meters, but only the center of the screen was used (Figure 1). 
The resulting image was 3.25 meters wide and 2.4 meters high, and had a resolution of $1400 \times 1050$ pixels. The physical field of view was $55^{\circ}$ horizontally and $45^{\circ}$ vertically. We used monoscopic rendering, with a frame rate of $50 \mathrm{~Hz}$. The projector used was a Barco Galaxy 7+.

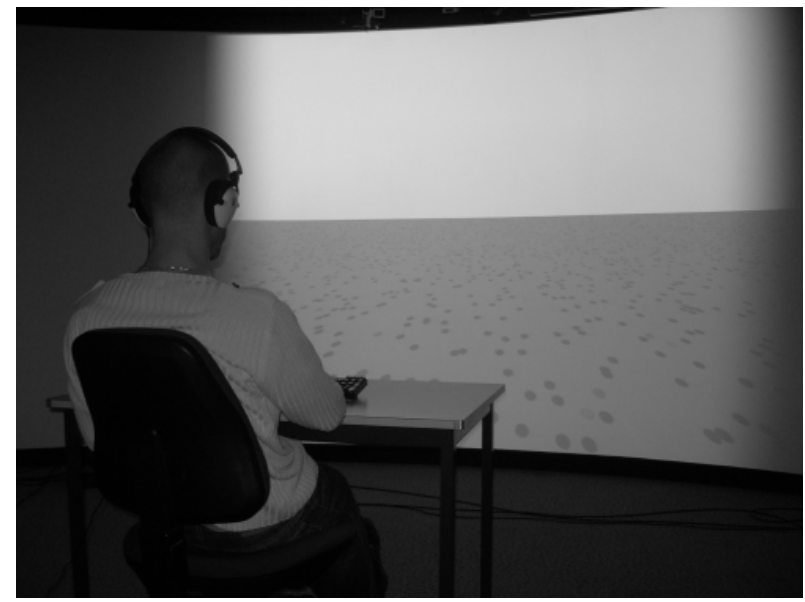

Figure 1: Experimental setup

The experiment was performed within an empty 3D virtual environment. All possible landmarks were removed so to avoid that the sizes of virtual objects give information about depths. The ground was made of a green color ( $4 \mathrm{db} 34 \mathrm{~d}$ in hexadecimal RGB format), and the sky of a gray/blue color (e6e6f2). In order to provide participants with visual flow during navigation, a set of 100000 gray points (4d804d) was displayed on the ground (see Figure 2). Each point had a radius of $5 \mathrm{~cm}$, was randomly positioned in the environment, and had a random lifetime of $5 \pm 3$ seconds. Using these limited lifetime dots, we avoided all problems of pattern repetitions peculiar to the use of textures.

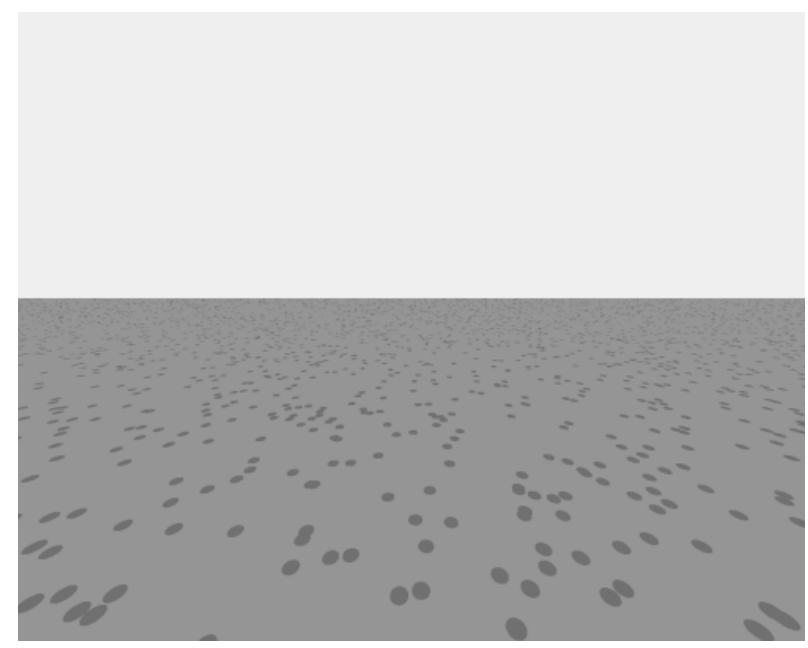

Figure 2: Visual display with random lifetime dots

\subsubsection{Distracting task}

In order to avoid time counting strategies when estimating traveled distance, participants had to complete a secondary task in parallel to the main task of estimating the traveled distance. During the passive navigation phase, they heard one to four random digits (from " 1 " to
"9"), directly spoken into headphones every $2 \pm 1$ seconds. The task was to memorize all the digits.

\subsubsection{Interaction}

Directly after the completion of each passive navigation, participants had to enter the digits they heard with the keyboard and then press the "Enter" key in order to move on to the reproduction phase. During the reproduction phase, participants used the "Up" key (forward translation) and the "Down" key (backward translation) to move through the VE. As long as they pressed the key, the camera continued to move in the given direction at a constant velocity $\left(V_{0}=1.2 \mathrm{~m} \cdot \mathrm{s}^{-1}\right)$. The motion stopped when the key was released. Note that there was a short acceleration and deceleration phase when the keys were pressed or released in order to avoid any strong discontinuity in the motion's speed. When participants estimated that they reproduced the correct distance, they validated their choice with the "Enter" key and the next trial started.

\subsubsection{Experimental plan}

The participants were exposed to two sets of 28 trials: one set for each motion condition, e.g., linear camera motion vs. oscillating camera motion.

- The linear camera motion was a linear translation of the camera at a constant speed $\left(V_{0}=1.2 \mathrm{~m} . \mathrm{s}^{-1}\right)$ as if the camera was on rails, or as if the user was driving a car.

- The oscillating camera motion was the same linear translation as in the linear camera motion $\left(V_{0}=1.2 \mathrm{~m} . \mathrm{s}^{-1}\right)$, but additionally, the camera position was slightly moved on the vertical, lateral and forward axes depending on time. The camera motion used corresponds exactly to the model proposed by Lécuyer et al. [5] and Hillaire et al. [3]. The main parameters of the camera motion were set to: $80 \mathrm{~cm}$ step length, $6 \mathrm{~cm}$ oscillation amplitude on the vertical axis and $2.5 \mathrm{~cm}$ oscillation amplitude on the two other axes. The period on the lateral axis was twice the period on the two other axes. These values correspond approximately to the physical values observed for a person of $1.70 \mathrm{~m}$ height. The focus point was constantly set to the center of the screen. This point was used to compute a compensation of the camera as explained in [5] and [3].

Participants were divided into 2 groups. One group $(n=6)$ was first presented with the Linear Camera Motion trials, while the other group $(n=6)$ was first presented with the Oscillating Camera Motion trials.

In each condition, the participants were exposed to 7 successive blocks of 4 trials with different distances $(5,7,10$ and 13 meters). In each block, the presentation order of these trials was randomized. During a learning phase, prior to each condition (linear or oscillating camera motion), participants were provided with a set of 4 trials that did not enter in the final data set. The entire experiment lasted approximately 30 minutes.

\subsection{Collected Data}

The traveled distance and the distance reproduced by the participants (in meters) were automatically recorded at the end of each trial. The digits heard and entered for the distracting task were also recorded.

At the end of the experiment, participants were asked to fill out a subjective questionnaire in which they had to explain which strategies they used to complete the task. Using a seven-point Likert scale they were asked to evaluate both modes of camera motion according to five criteria: (1) perception of distances, (2) immersion feeling, (3) impression of realism, (4) sensation of walking, and finally (5) simulator sickness. 


\subsection{Results}

Two different error measures were analyzed: the signed distance error and the absolute distance error. The signed distance error corresponds to the reproduced distance minus the required distance. The absolute distance error corresponds to the absolute value of the reproduced distance minus the required distance.

\subsubsection{Signed Distance Errors}

The participants' performance in reproducing the navigated distance is characterized by an overall tendency to undershoot the distances (see Figure 3(a) and Figure 3(b)). Indeed, among the 12 participants, 8 participants undershot distances for all conditions and distances. Two participants overshot distances only for short distances and only for the linear camera motion. One participant overshot distances for all distances and only for the linear camera motion. Last, only one participant overshot all the distances for both conditions.

An ANOVA on signed distance errors (factors: navigated distance $[5 \mathrm{~m}, 7 \mathrm{~m}, 10 \mathrm{~m}, 13 \mathrm{~m}]$ and camera motion [oscillating, linear]) revealed a significant effect of navigated distance $(F(1)=19.0$, $p \ll .001)$. This effect reflects that the participants' tendency to undershoot the required distance increased with the length of the traveled distance.

Moreover, as illustrated on Figure 3(a) vs. Figure 3(b), there appear to be more outliers and more overshootings with the linear camera motion than with the oscillating camera motion. This suggests that the use of oscillating camera motions can reduce variance and increase global accuracy. Indeed, for short distances $(5 \mathrm{~m}$ and $7 \mathrm{~m}$ ), the standard deviations of participants' signed responses were found significantly smaller in the oscillating camera motion condition $(0.83)$ than in the linear camera motion condition (1.27) $(t(11)=2.71, p=.02)$. For long distances $(10 \mathrm{~m}$ and $13 \mathrm{~m})$ the standard deviations of participants' signed responses did not differ significantly (linear motion condition: 1.60; oscillating condition: 1.38 ; $\mathrm{t}(11)=.98, \mathrm{p}=.35)$.

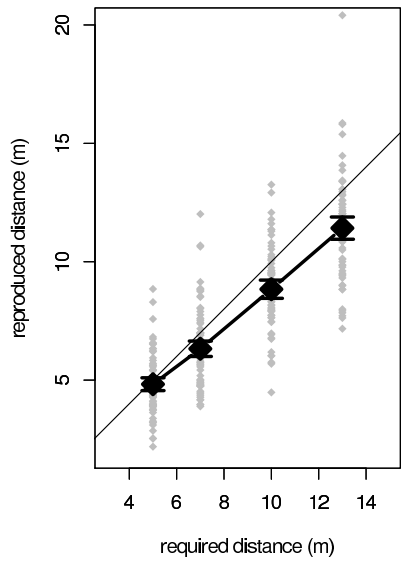

(a) Linear camera motions

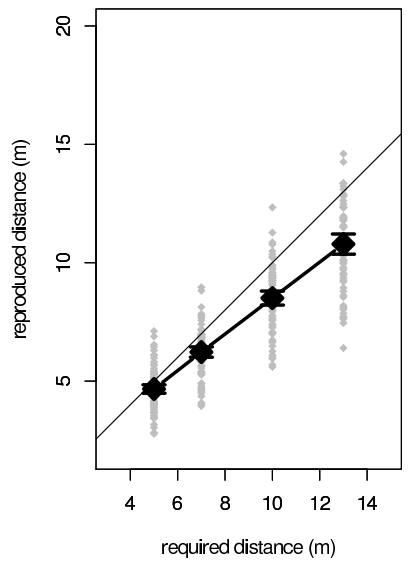

(b) Oscillating camera motions
Figure 3: Distances reproduced by the participants vs. Required distances for both camera conditions

\subsubsection{Absolute Distance Errors}

An ANOVA (factors: navigated distance [5m, 7m, 10m, 13m] and camera motion [oscillating, linear]) revealed a significant effect of navigated distance $(F(3)=16.58, p<.001)$ as well as a significant interaction between navigated distance and camera motion $(F(3)=3.02, p<.05)$. To further characterize the nature of the interaction we pooled data over short distances $(5 \mathrm{~m}$ and $7 \mathrm{~m})$ and over long distances (10m and $13 \mathrm{~m})$ and analyzed the data again (see Figure 4). A T-test revealed a marginally significant difference for short distances between the oscillating and linear camera motion $(t(11)=2.05, p=.065)$. Together with the significant interaction described above, and the reduction in variability, this tendency suggests that distance reproduction performance for short distances is more accurate with oscillating camera motion than with linear camera motion. For long distances, however, both kinds of camera motion rendered similar results.

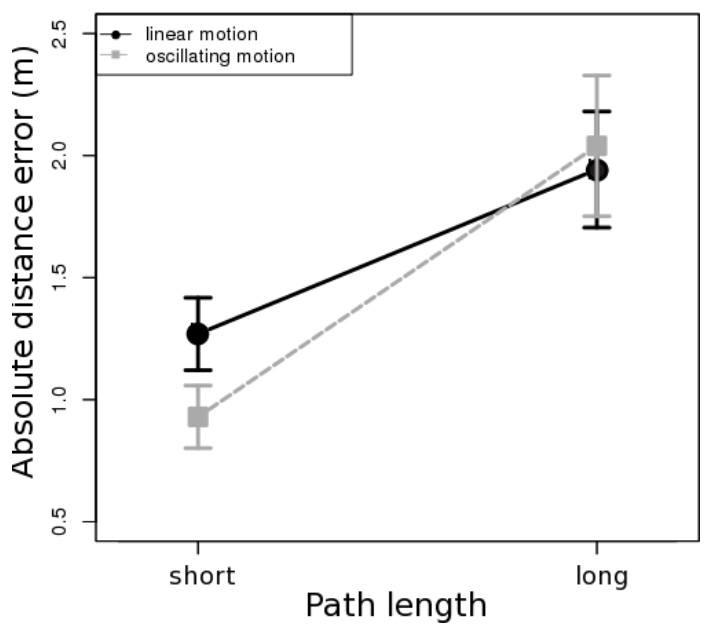

Figure 4: Absolute distance errors for short distances $(5 \mathrm{~m}, 7 \mathrm{~m})$ and long distances $(10 \mathrm{~m}, 13 \mathrm{~m})$

\subsubsection{Questionnaire}

The results of the subjective questionnaire confirmed the results of Lécuyer et al. [5]. A Wilcoxon signed rank test showed significant differences for distance perception $(z=-2.17, p=.03)$, walking sensation $(z=-2.68, p<.01)$ and a marginally significant difference for realism $(z=-1.77, p=.08)$. For these three criteria, on average participants seem to have preferred the oscillating camera motions (see Figure 5). However, the criteria of immersion and simulator sickness failed to be significant.

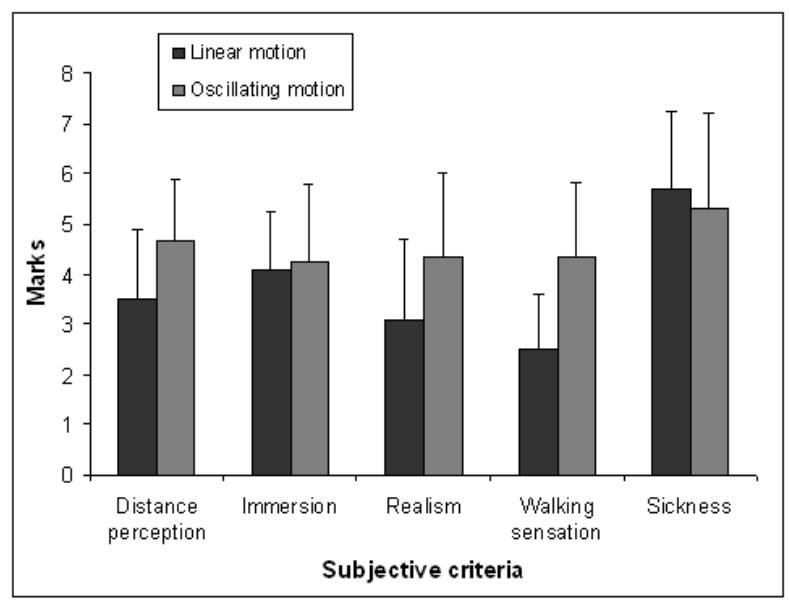

Figure 5: Subjective evaluation of oscillating camera motion vs. linear camera motion

The participants were also asked to explain the strategies they used in order to complete the main task. Eight reported to have 
counted the number of steps during the oscillating motion, even if the secondary task made it complicated. In addition to this main strategy, five of them also tried to remember or "feel" the elapsed time for the linear motion or for both motions. Five participants reported to have tried to memorize the "rhythm" of occurrence of the digits of the secondary task, even if the digits appeared randomly. Three of the participants stated that they used a strategy involving the visual flow.

\section{Discussion}

Results from this study suggest that the oscillating camera motion increases the accuracy of the perception of traveled distance, at least for short distances. This is supported by the significant decrease in standard deviations of participants' responses and the marginally significant decrease of absolute distance error values, in the oscillating camera motion condition and for short distances.

Participants globally undershot traveled distances. Undershooting distances is a result that is often found and discussed in the literature [7] [4]. However, it is sometimes found that participants overshoot traveled distances, such as in [8]. The main differences between our experiment and experiments found in related work (e.g., Riecke et al. [8]) concern the generated visual flow and the field of view. For instance, the field of view used in our setup is smaller than the one used in Riecke et al. [8].

The difference in performance concerning the estimation of traveled distance between oscillating camera motion and linear camera motion is found to be marginally significant. This marginality might be due to the secondary task used. This secondary task is a classical distracting task in experimental studies on distance perception which avoids that participants count the elapsed time. But it also prevent them counting the number of steps with the oscillating camera motion. It is possible that without the secondary task, the accuracy of the reproduced distances could have been increased with the oscillating camera motions. Indeed, if participants would have been able to count precisely the number of steps, they would have been able to reproduce the distances very accurately by reproducing the same number of steps. Moreover, we found that the effect of camera condition was marginally significant only for short distances. One explanation could be that our secondary task was more difficult for the long paths. Indeed, the number of digits that participants had to remember increased with the length of the path. Thus, the complexity increased and it presumably introduced more variance for long paths.

It could also be interesting to conduct this experiment again with an eye-tracking system in order to adapt the oscillating camera motion to the point on screen the user is actually looking at, such as in [3]. This implementation has been subjectively preferred by participants in [3]. Thus, it might also improve the perception of traveled distance.

The results of our subjective questionnaires confirmed some of the results found in [5] and [3]. Our questionnaires showed that participants preferred the compensated oscillating camera motion over the linear camera motion. They found that oscillating camera motions increased both the distance perception and the walking sensation. Besides, the subjective questionnaires showed no significant differences between the oscillating camera motion and the linear camera motion for the immersion criterion. It is maybe a consequence of the limited field of view, or of the visual environment that was not really reallistic (random lifetime dots without other landmarks). The simulator sickness criterion also failed to be significant. One explanation could be the poor diversity of traveled paths. Indeed, traveled paths were only straight lines. Participants never had to turn or make complex trajectories. More complex paths could make them feel more dizzy. Another explanation is that the random lifetime dots seem to have disturbed the participants more than what we expected. The resulting visual flow could be tiring for the eyes and it might have had a high influence on the simulator sickness criterion, more than the camera motion condition.

\section{Conclusion}

This paper investigated the use of camera motion to improve the perception of traveled distances in VE. We found that the accuracy of the reproduced distances seems to be increased by compensated oscillating camera motions, at least for short traveled distances. Taken together, our results suggest a positive influence of the camera motions on the perception of distances and on the sensation of walking in VE.

Future work will investigate the influence of camera motions on the perception of distances for more complex paths, on the sense of orientation, and on the perception of other properties of virtual environents such as scale. We will also study whether dynamically adapting the focus point by measuring participants' gaze during navigation results in similar or even increased path integration performance.

\section{ACKNOWLEDGEMENTS}

The research leading to these results has received funding from the European Community's Seventh Framework Programme under FET-Open grant agreement $n^{\circ} 222107$ NIW - Natural Interactive Walking, and the Volkswagen Foundation.

\section{REFERENCES}

[1] L. R. Harris, M. Jenkin, and D. C. Zikovitz. Vestibular cues and virtual environments: Choosing the magnitude of the vestibular cue. In Proceedings of the IEEE conference on Virtual Reality, pages 229-236, 1999.

[2] L. R. Harris, M. Jenkin, D. C. Zikovitz, F. Redlick, P. M. Jaekl, U. Jasiobedzka, H. L. Jenkin, and R. S. Allison. Simulating self-motion I: Cues for the perception of motion. Virtual Reality, 6(2):75-85, 2002.

[3] S. Hillaire, A. Lécuyer, R. Cozot, and G. Casiez. Using an eyetracking system to improve camera motions and depth-of-field blur effects in virtual environments. In Proceedings of the IEEE conference on Virtual Reality, pages 47-50, 2008.

[4] V. Interrante, B. Ries, and L. Anderson. Distance perception in immersive virtual environments, revisited. In Proceedings of the IEEE conference on Virtual Reality, pages 3-10, 2006.

[5] A. Lécuyer, J.-M. Burkhardt, J.-M. Henaff, and S. Donikian. Camera motions improve the sensation of walking in virtual environments. In Proceedings of the IEEE conference on Virtual Reality, pages 11-18, 2006.

[6] T. Meilinger, M. Knauff, and H. H. Bülthoff. Working memory in wayfinding - a dual task experiment in a virtual city. Cognitive Science: A Multidisciplinary Journal, 32(4):755-770, 2008.

[7] J. M. Plumert, J. K. Kearney, and J. F. Cremer. Distance perception in real and virtual environments. In Proceedings of the 1st Symposium on Applied perception in graphics and visualization, pages 27-34, 2004.

[8] B. E. Riecke, J. Schulte-Pelkum, and H. H. Bülthoff. Perceiving simulated ego-motions in virtual reality - comparing large screen displays with hmds. Proceedings of the SPIE, 5666:344-355, 2005.

[9] B. E. Riecke, H. A. van Veen, and H. H. Bülthoff. Visual homing is possible without landmarks: A path integration study in virtual reality. Presence: Teleoperators and Virtual Environments, 11(5):443-473, 2002.

[10] B. E. Riecke and J. M. Wiener. Can people not tell left from right in VR? point-to-origin studies revealed qualitative errors in visual path integration. In Proceedings of the IEEE conference on Virtual Reality, pages 3-10, 2007.

[11] J. M. Wiener and H. A. Mallot. Path complexity does not impair visual path integration. Spatial Cognition and Computation, 6(4):333-346, 2006. 